\title{
Extracorporeal Oxygenation Techniques in Adult Critical Airway Obstruction: A Review
}

\author{
Michal Pořízka ${ }^{1}$, Pavel Michálek ${ }^{1,2}$, Jiří Votruba ${ }^{3}$, Basem B. Abdelmalak $^{4}$ \\ ${ }^{1}$ Department of Anesthesiology and Intensive Care Medicine, First Faculty \\ of Medicine, Charles University and General University Hospital in Prague, Prague, \\ Czech Republic; \\ ${ }^{2}$ Department of Anaesthesia, Antrim Area Hospital, Antrim, United Kingdom; \\ ${ }^{3} 1^{\text {st }}$ Department of Tuberculosis and Respiratory Diseases, First Faculty \\ of Medicine, Charles University and General University Hospital in Prague, Prague, \\ Czech Republic; \\ ${ }^{4}$ Departments of General Anesthesiology and Outcomes Research, Cleveland Clinic, \\ Cleveland Clinic Lerner College of Medicine of Case Western Reserve University, \\ Cleveland, USA
}

Received June 22, 2020; Accepted April 30, 2021.

Key words: Extracorporeal circulation - Extracorporeal membrane oxygenation ECMO - Airway surgery - Respiratory insufficiency - Airway obstruction

Abstract: Extracorporeal life support has been increasingly utilized in different clinical settings to manage either critical respiratory or heart failure. Complex airway surgery with significant or even total perioperative airway obstruction represents an indication for this technique to prevent/overcome a critical period of severe hypoxaemia, hypoventilation, and/or apnea. This review summarizes the current published scientific evidence on the utility of extracorporeal respiratory support in airway obstruction associated with hypoxaemia, describes the available methods, their clinical indications, and possible limitations. Extracorporeal membrane oxygenation using veno-arterial or veno-venous mode is most commonly employed in such scenarios caused by endoluminal, external, or combined obstruction of the trachea and main bronchi.

Mailing Address: Michal Pořízka, MD., PhD., E.D.I.C., Department of Anesthesiology and Intensive Care Medicine, First Faculty of Medicine, Charles University and General University Hospital in Prague, U Nemocnice 2, 12808 Prague 2, Czech Republic; Mobile Phone: +420 702089 475; e-mail: michal.porizka@vfn.cz 


\section{Introduction}

A complex airway surgery includes a spectrum of interventional bronchological, otorhinolaryngological, and thoracic surgical procedures for various malignant and benign lesions of the upper airway and tracheobronchial tree. The requirements for the unobstructed surgical field and sufficient oxygenation with ventilation represent a challenging task for both surgeon and anaesthetist (Abdelmalak and Doyle, 2020). A majority of these procedures can be successfully and safely performed using a variety of ventilation techniques including intermittent apnea, jet ventilation (Pearson and McGuire, 2017) or high flow nasal oxygenation (HFNO). However, in cases with significant or even total airway obstruction, these methods are not sufficient in providing adequate respiratory support with the risk of developing severe and life-threatening hypoxemia and hypercapnia. Furthermore, following reconstructive surgery on the tracheobronchial tree, the suture line or the anastomosis site may be at high risk of dehiscence or disruption due to the endotracheal tube presence or pressures exerted by mechanical ventilation (Auchincloss and Wright, 2016). In such circumstances, extracorporeal life support devices including cardio-pulmonary bypass, extracorporeal membrane oxygenation, and pumpless lung assist devices should be utilized.

\section{Methodology}

A comprehensive electronic and manual search of databases PubMed, Web of Science, and Scopus was performed for a period from January 1980 till April 2020 using the following terms: "extracorporeal oxygenation", "airway surgery", "airway obstruction", "tracheal surgery". The following types of articles were retrieved: randomized controlled trials, prospective and retrospective cohort studies, case reports, and reviews.

\section{Cardio-pulmonary bypass}

Historically, the first documented use of conventional cardio-pulmonary bypass (CPB) for airway surgery was published by Woods et al. in 1961 and this technique was further used by many centers worldwide (Tyagi et al., 2006). The main advantage of CPB is intraoperative haemodynamic stability and a possibility of "recycling" surgical field suctioned blood returning it back to the circuit, thus significantly limiting the total intraoperative blood loss (Pillai and Suri, 2008). On the other hand, the necessity for high level of anticoagulation with heparin during the procedure, and serious CPB-related sequelae such as induction of postoperative coagulopathy, development of systemic inflammatory response, acute renal failure or acute lung injury has resulted in the development of less invasive methods (Pillai and Suri, 2008).

\section{Extracorporeal membrane oxygenation}

Extracorporeal membrane oxygenation (ECMO) is a modified cardio-pulmonary bypass machine providing extracorporeal decarboxylation and oxygenation. It can be 


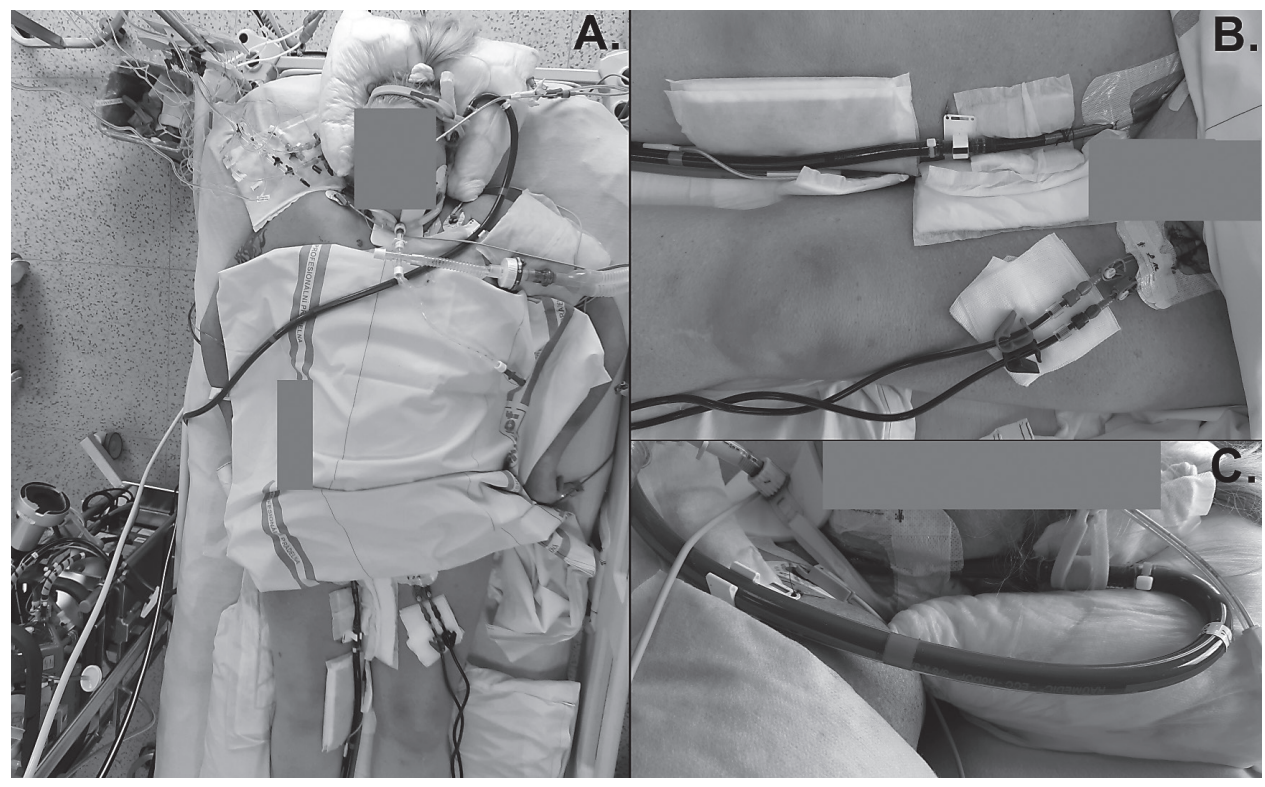

Figure 1 - A. Photograph showing VV ECMO (veno-venous extracorporeal membrane oxygenation) (Cardiohelp, Getinge, Sweden) in a patient with severe influenza pneumonitis; $B$. A detail of the drainage cannula in the right femoral vein; C. A detail of the return cannula in the left internal jugular vein (patient's written consent for publication of these images obtained in 2018).

configurated in a veno-venous ( $\mathrm{VV}$ ) used for the management of critical respiratory failure, or in a veno-arterial (VA) setting providing both respiratory and cardiac support (Fierro et al., 2018). VV ECMO (Figure 1) can be instituted peripherally using the two cannulas (femoro-jugular, femoro-femoral) or with the double-lumen cannula inserted under the ultrasound and echocardiographic guidance via the internal jugular vein. VA ECMO can be cannulated peripherally by a transcutaneous Seldinger technique into the femoral vessels or centrally using a surgical approach via median sternotomy into the right atrium and ascending aorta (Fierro et al., 2018). Most ECMO cannulations are carried out after induction of general anaesthesia, however in cases of anticipated high likelihood of losing the airway during a complicated endotracheal intubation, awake cannulation can be safely performed (Gardes and Straker, 2012). When used for this indication, patients are usually weaned off and disconnected from ECMO at the end of surgical procedure or within the immediate postoperative period, when definite airway access is secured and native lung function has proven adequate (Lang et al., 2015). However, in cases of unresolved pulmonary pathology with ongoing respiratory insufficiency or a significant haemodynamic instability, ECMO has to be continued and subsequently weaned off gradually in the postoperative period as the underlying condition improves. 
The current scientific evidence on ECMO use in high-risk airway surgery is based only on case reports and small case series. Initially, most of the reports came from the paediatric population. ECMO has been successfully used in children undergoing invasive procedures for foreign body related critical airway obstruction (Park et al., 2014), congenital tracheal stenosis (Kunisaki et al., 2008) and malignant or benign endotracheal masses (Smith et al., 2009). Children, and especially infants are extremely prone to serious airway obstruction due to the already narrowed subglottic diameter of the trachea. Thus, according to Hagen-Pouiselle's law, even a small airway narrowing of any cause can lead to critical airway obstruction and respiratory arrest (Harless et al., 2014). In adults, the first use of ECMO in a patient undergoing tracheal resection was published by Onozawa et al. (1999). Since then, more adult ECMO experience has been increasingly reported. ECMO has been reliably used in a variety of high-risk airway surgical and endoscopic procedures in patients presenting with airway and thoracic tumours (Lang et al., 2015), trauma (Yuan et al., 2008), intrinsic obstruction (Jeon et al., 2006) or extrinsic compression syndromes (De Piero et al., 2018).

The important advantage of ECMO compared to CPB is the minimal spread of tumour cells in oncological airway surgery. The suction of blood from the surgical field into a CPB reservoir creates a risk for disseminating cancer cells into remote organs. Such a case was published by Hasegawa et al. (2002), who reported the pulmonary dissemination of thyroid cancer cells after a CPB-assisted thyroidectomy. As ECMO consists of a closed circuit without a reservoir, no blood from the surgical field is reinfused into the patient and therefore such risk is negligible. Alternatively, the suctioned blood from the field should be processed by a cell saver machine, and reinfused to the patient after filter through a leukocyte depletion filter that's known to filter out cancer cells because of their negative electrical charge (Gwak et al., 2005).

\section{Veno-venous ECMO}

By providing an adequate gas exchange, $\mathrm{VV}$ ECMO can enable extensive airway surgical interventions with superior visualisation and access to the surgical field. As a sole respiratory support, VV ECMO is fully sufficient in most cases having the advantage of less invasive cannulation and reduced risk of mechanical and bleeding complications compared to VA ECMO with arterial cannulation (Guglin et al., 2019). Venous drainage cannulas of 21-27 Fr in femoral position, return cannulas of 17-23 Fr in femoral or jugular position or 27-31 Fr jugular double-lumen cannulas are commonly used in adult patients to provide blood flow of $50-100 \mathrm{ml} / \mathrm{kg}$. Inspired oxygen fraction $\left(\mathrm{FiO}_{2}\right)$ and sweep gas flow in the ECMO oxygenator are tailored to achieve proper gas exchange with physiologic values of $\mathrm{PaO}_{2}$ and $\mathrm{PaCO}_{2}$ in the arterial blood gas analysis (Sen et al., 2016). The most common complication of VV ECMO is post-cannulation venous thrombosis that can be present in up to $62 \%$ of cases of prolonged ECMO (Fisser et al., 2019). However, due to the short duration 
of ECMO in airway surgical patients, such complication is likely to be minimal and has not been reported so far.

\section{Veno-arterial ECMO}

VA ECMO should be used in cases with supposed or already ongoing haemodynamic instability including impending cardiac arrest (Willms et al., 2012). There have been several studies published, reporting the feasibility of VA ECMO use in patients undergoing complex oncological airway or goitre surgery (Shao et al., 2009). It may be technically difficult to cannulate ECMO in the emergency settings including respiratory or cardiac arrest situations. In such circumstances, femoro-femoral cannulation in the sitting or semirecumbent position is the method of choice and successful cases of such approach have been reported (Kim et al., 2015). Arterial cannulas of 15-21 Fr and drainage venous cannulas of 21-27 Fr should provide sufficient ECMO blood flow of $50-70 \mathrm{ml} / \mathrm{kg} / \mathrm{min}$, fully substituting the patient's own cardiac output. Extensive monitoring during VA ECMO generally includes invasive arterial blood pressure, evaluation of oxygen delivery adequacy by measuring arterial lactate and mixed central venous oxygen hemoglobin saturation, and possibly tissue oximetry (Guglin et al., 2019). Limb ischaemia is a frequent complication of peripheral VA ECMO; however, its incidence has been markedly reduced by the routine use of the distal limb reperfusion with 6-8 Fr antegrade catheter inserted into the superficial femoral artery (Guglin et al., 2019).

\section{Pumpless lung assist}

The third option for extracorporeal respiratory support during complex airway surgery represents the use of pumpless extracorporeal lung-assist devices (lung membrane). These are based on extracorporeal $\mathrm{CO}_{2}$ removal with only limited oxygenation capacity using low transmembrane gradient oxygenators with blood flow driven by the patient's own cardiac output (Walles, 2007). Typical cannulation sites include femoral artery and vein, however, cases with central cannulation via thoracotomy used for the treatment of pulmonary hypertension have been reported. The main disadvantages of this method compared to ECMO are the limited gas exchange capacity, dependence on adequate cardiac output, and inability of hemodynamic support if needed (Walles, 2007). However, potential benefits of the membrane lung include a reduction in cellular trauma and minimal inflammatory activation compared to ECMO and CPB (Iglesias et al., 2008). There is only anecdotal experience with this method.

\section{Anticoagulation and fluid management in ECMO}

A need for full anticoagulation represents a major drawback of using CPB for airway surgery. ECMO with heparin-coated cannullae and circuit tubings requires a much lower level of heparinization, usually aiming for activated clotting time of 160-180 s, thus offering the advantage of less bleeding complications. For 
instance, in a recent study, no bleeding complications and no more than the usual intraoperative blood loss were observed in 10 patients undergoing complex tracheobronchial surgery on VA ECMO with only minimal anticoagulation of 3,000-5,000 units of heparin (Lang et al., 2015). Furthermore, in another study, no anticoagulation at all was used in a patient on VV ECMO undergoing tracheal surgery without any thrombotic events (Antonacci et al., 2018). Usually, at the end of the airway procedure, protamine is administered for heparin anticoagulation effect reversal and prevention of postoperative bleeding. Afterwards, ECMO cannulas can be removed with manual compression and skin suture. In cases of complicated arterial cannulation, a vascular surgical revision and repair may be required. ECMO requires a smaller amount of circuit priming compared to $\mathrm{CPB}$, thus the risk of dilutional coagulopathy and volume overload associated complications including pulmonary oedema may be reduced (Wiebe et al., 2006).

\section{Immunosuppressive effects of ECMO}

The theoretical disadvantage of extracorporeal methods lies in the temporary immunosupressive effect caused by the increases in immunoregulatory factors including interleukin-10, tumor necrosis factor, and transforming growth factor- $\beta$. This may cause changes in the function of the immune system and cancer surveillance, possibly leading to the spread and growth of cancer cells (Sablotzki et al., 1997). This is supported by the study, in which a trend towards the progression of cancer was observed in patients undergoing cardiac surgery with the use of CPB compared to off-pump surgery (Pinto et al., 2013). On the other hand, ECMO is a mini-invasive extracorporeal method comparable to miniaturized CPB systems, which have been shown to have a diminished immune reaction (Vohra et al., 2009). Therefore, the effect of ECMO on potential cancer growth is likely to be insignificant and has not been documented in the literature so far.

\section{Complications of ECMO}

Generally, there are many mechanical, haemorrhagic, thromboembolic and infectious complications associated with the use of ECMO described in critically ill patients requiring lung or heart support. All of these seriously affect the patient's outcome, increasing both morbidity and mortality (Marasco et al., 2008). Mechanical problems, represented by oxygenator or pump failure, circuit thrombosis, cannulae-associated, and limb ischaemic complications require multidisciplinary approach including intensive care physicians, vascular surgeons, cardiologists, and perfusionists. Nevertheless, patients undergoing airway surgery with short ECMO runs are likely to have a much lower incidence of such serious complications compared to the critically ill, and to our knowledge, they have not been reported yet.

One case series reported successful use of extracorporeal lung membrane in combination with intermittent apnoeic ventilation in 15 patients undergoing 


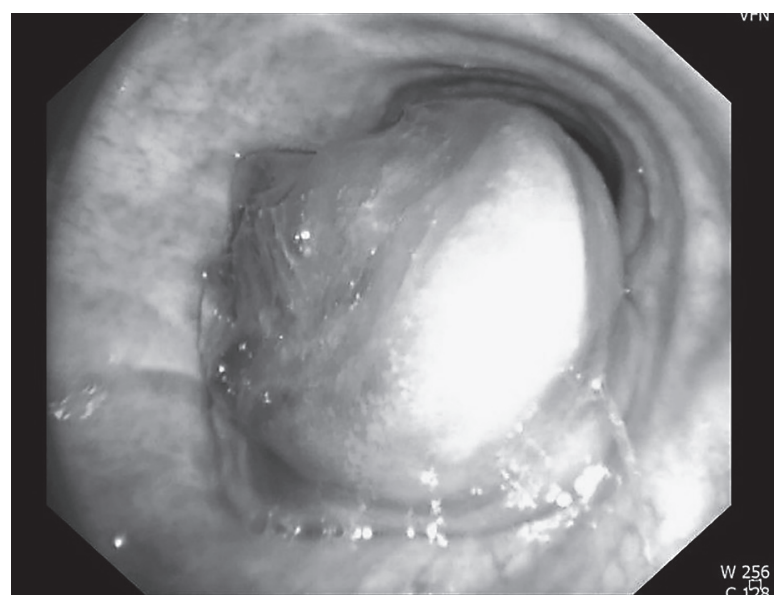

Figure 2 - Endoluminal tracheal tumour causing almost complete obstruction of the airway passage.

complex airway surgery without any complications and with minimal effect on coagulation and inflammatory response (Sanchez-Lorente et al., 2012).

\section{Extracorporeal oxygenation in tracheal reconstructions}

A potential advantage of extracorporeal techniques in reconstructive procedures on trachea (Figure 2) is the allowance of stable surgical field without risks of hypoxemic adverse events. Most case reports are related to new-born or toddler reconstructions due to congenital malformations of the trachea. Three larger case series report the use of ECMO in 32 adult patients undergoing open tracheal resections or extended reconstructions of the tracheobronchial tree (Chang et al., 2014; Kim et al., 2015; Lang et al., 2015). While two former studies employed the VA ECMO circuit in all cases, Kim et al. (2015) have used mostly VV ECMO. Only one patient died within 30 days after surgery due to multiorgan system failure, all remaining patients were successfully discharged. Another retrospective study reported a cohort of 18 patients with the central airway obstruction syndrome caused by malignant conditions (Hong et al., 2013). These patients had a VV ECMO support as a bridge before the procedure of interventional bronchology aiming to restore patency of the airways. Five of these patients (27.8\%) died at 60 days.

\section{Extracorporeal oxygenation in the external airway compression}

An anterior mediastinal mass (AMM) (Figure 3), for example, lymphomas, retrosternal goitre or thymoma may result in a life-threatening external airway compression in the intraoperative period. $\mathrm{V}$ ECMO has been reported in several cases of the significant external lower trachea or main bronchi obstruction, as planned femoral vessel cannulation at the start of the procedure (Goh et al., 1999) or as a rescue technique in sudden ventilation and oxygenation difficulties during a lymphatic node excision (Netri et al., 2016). This technique may also be used awake, 


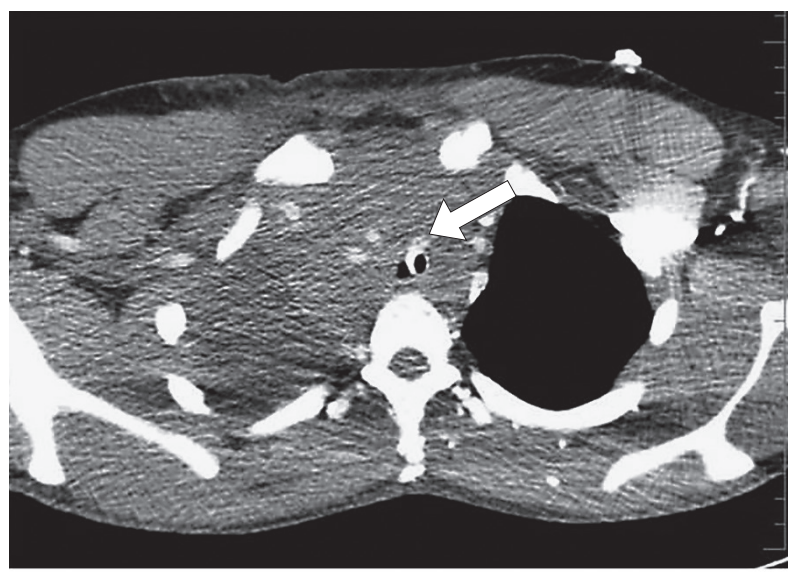

Figure 3 - An extensive anterior mediastinal mass causing external compression of distal trachea and main bronchi.

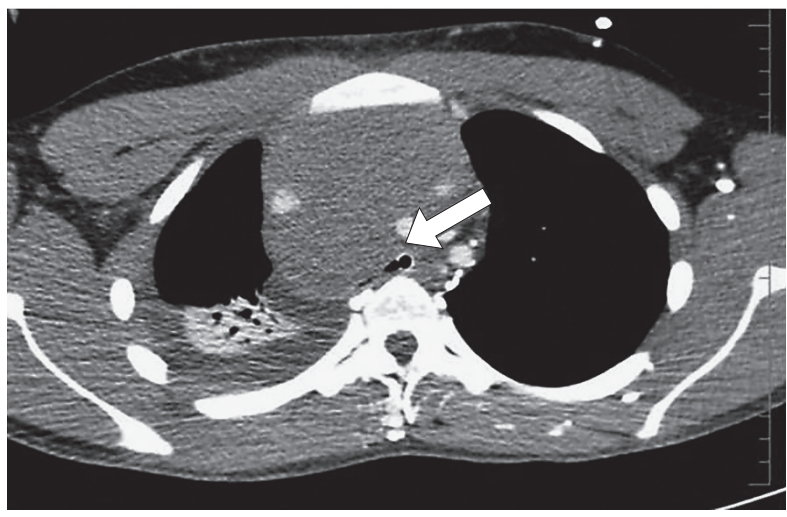

as a respiratory support tool, in patients undergoing chemotherapy for extensive intrathoracic lymphatic masses causing significant breathing problems (Worku et al., 2015). It should be noted that such approach may not be necessary at all in low and moderate risk AMM cases, however it should be entertained in the high risk AMM situations causing severe symptoms and $>50 \%$ tracheal compression. Even in severe cases conservative approach with awake intubation, and gradual deepening of the anesthetic preserving spontaneous ventilation has proved successful in the past (Abdelmalak et al., 2010). Clinicians must keep in mind that having CPB, or ECMO equipment on stand-by may not always ensure a favorable outcome (Slinger and Karsli, 2007). Despite the immediate availability of the ECMO team, it may take approximately 5-10 minutes to achieve adequate oxygenation after complete airway obstruction (Tempe et al., 2001). This delay might pose a risk of hypoxic brain injury. Therefore, a well thought out anesthetic plan that allows for maintaining the airway and ventilation throughout lessens the chances of the need for such an invasive intervention. 


\section{Extracorporeal oxygenation in and whole lung lavage}

Many case reports described the use of ECMO in whole lung lavage procedure for pulmonary alveolar protenosis (PAP) (Sivitanidis et al., 1999). However, that option should be considered carefully in light of the known potential complications of ECMO, and also the fact that this procedure can be performed effectively even in the severe cases of PAP in a sequential manner in centers with extensive experience in such procedures (Abdelmalak et al., 2015).

\section{Awake extracorporeal oxygenation}

Extracorporeal oxygenation has been used in one case of awake video-assisted thoracic surgery, in the patient with advanced chronic obstructive pulmonary disease, emphysema, and hypoxaemia at rest (Drosos et al., 2020). Although awake tracheal reconstructions have also been reported in the literature, the use of the extracorporeal oxygenation support while awake has been limited only to the "suture sparing" strategy of tracheal reconstruction during the immediate postoperative period (Schieren et al., 2017).

\section{Conclusion}

In conclusion, extracorporeal respiratory support represents the method of choice in patients undergoing complex high-risk airway procedures with the risk of profound hypoxaemia and hypercapnia due to significant intraluminal, external or mixed airway obstruction. Given the risk to benefit ratio, ECMO, either in $\mathrm{VV}$ or VA mode is the most viable method providing adequate oxygenation and ventilation support with the acceptable risk of complications. However, it has to be mentioned, that the techniques of extracorporeal oxygenation must be accompanied by other methods of restoring the airways such as tracheal or bronchial dilation and stenting, the mass removal or debulking, or surgical reconstruction of the airways.

\section{References}

Abdelmalak, B. B., Doyle, D. J. (2020) Recent trends in airway management. F1000Res. 9, 355.

Abdelmalak, B. B., Marcanthony, N., Abdelmalak, J., Machuzak, M. S., Gildea, T. R., Doyle, D. J. (2010) Dexmedetomidine for anesthetic management of anterior mediastinal mass. J. Anesth. 24(4), 607-610.

Abdelmalak, B. B., Khanna, A. K., Culver, D. A., Popovich, M. J. (2015) Therapeutic whole-lung lavage for pulmonary alveolar proteinosis: a procedural update. J. Bronchology Interv. Pulmonol. 22(3), 251-258.

Antonacci, F., De Tisi, C., Donadoni, I., Maurelli, M., lotti, G., Taccone, F. S., Orlandoni, G., Pellegrini, C., Belliato, M. (2018) Veno-venous ECMO during surgical repair of tracheal perforation: a case report. Int. J. Surg. Case Rep. 42, 64-66.

Auchincloss, H. G., Wright, C. D. (2016) Complications after tracheal resection and reconstruction: prevention and treatment. J. Thorac. Dis. 8, S160-S167.

Chang, X., Zhang, X., Li, X., Xu, M., Zhao, H., Fang, W., Yao, F. (2014) Use of extracorporeal membrane oxygenation in tracheal surgery: a case series. Perfusion 29(2), 159-162.

De Piero, M. E., Fontana, D., Quaglino, F., Attisani, M., Baroncelli, F., Cavallo, A., Gentile, T., Livigni, S. (2018) 
Extracorporeal membrane oxygenation (ECMO)-assisted surgery for mediastinal goiter removal. J. Cardiothorac. Vasc. Anesth. 32(1), 448-451.

Drosos, V., Kersten, A., Spillner, J., Kalverkamp, S. (2020) Awake thoracic surgery with extracorporeal membrane oxygenation. Surg. Case Rep.

Fierro, M. A., Daneshmand, M. A., Bartz, R. R. (2018) Perioperative management of the adult patient on venovenous extracorporeal membrane oxygenation requiring noncardiac surgery. Anesthesiology 128(1), $181-201$.

Fisser, C., Reichenbächer, C., Müller, T., Schneckenpointner, R., Malfertheiner, M. V., Philipp, A., Foltan, M., Lunz, D., Zeman, F., Lubnow, M. (2019) Incidence and risk factors for cannula-related venous thrombosis after venovenous extracorporeal membrane oxygenation in adult patients with acute respiratory failure. Crit. Care Med. 47(4), e332-e339.

Gardes, J., Straker, T. (2012) Impossible airway requiring venovenous bypass for tracheostomy. Case Rep. Anesthesiol. 2012, 592198.

Goh, M. H., Liu, X. Y., Goh, Y. S. (1999) Anterior mediastinal masses: an anaesthetic challenge. Anaesthesia 54, 670-674.

Guglin, M., Zucker, M. J., Bazan, V. M., Bozkurt, B., El Banayosy, A., Estep, J. D., Gurley, J., Nelson, K., Malyala, R., Panjrath, G. S., Zwischenberger, J. B., Pinney, S. P. (2019) Venoarterial ECMO for adults: JACC Scientific Expert Panel. J. Am. Coll. Cardiol. 73(6), 698-716.

Gwak, M. S., Lee, K. W., Kim, S. Y., Lee, J., Joh, J. W., Kim, S. J., Lee, H. H., Park, J. W., Kim, G. S., Lee, S. K. (2005) Can a leukocyte depletion filter (LDF) reduce the risk of reintroduction of hepatocellular carcinoma cells? Liver Transpl. 11(3), 331-335.

Harless, J., Ramaiah, R., Bhananker, S. M. (2014) Pediatric airway management. Int. J. Crit. Illn. Inj. Sci. 4(1), 65-70.

Hasegawa, S., Otake, Y., Bando, T., Cho, H., Inui, K., Wada, H. (2002) Pulmonary dissemination of tumor cells after extended resection of thyroid carcinoma with cardiopulmonary bypass. J. Thorac. Cardiovasc. Surg. 124, 635-636.

Hong, Y., Jo, K. W., Lyu, J., Huh, J. W., Hong, S. B., Jung, S. H., Kim, J. H., Choi, C. M. (2013) Use of venovenous extracorporeal membrane oxygenation in central airway obstruction to facilitate interventions leading to definitive airway security. J. Crit. Care 28, 669-674.

Iglesias, M., Jungebluth, P., Petit, C., Matute, M. P., Rovira, I., Martínez, E., Catalan, M., Ramirez, J., Macchiarini, P. (2008) Extracorporeal lung membrane provides better lung protection than conventional treatment for severe postpneumonectomy noncardiogenic acute respiratory distress syndrome. J. Thorac. Cardiovasc. Surg. 135(6), 1362-1371.

Jeon, K., Kim, H., Yu, C. M., Koh, W. J., Suh, G. Y., Chung, M. P., Kwon, O. J. (2006) Rigid bronchoscopic intervention in patients with respiratory failure caused by malignant central airway obstruction. J. Thorac. Oncol. 1(4), 319-323.

Kim, C. W., Kim, D. H., Son, B. S., Cho, J. S., Kim, Y. D., I, H., Ahn, H. Y. (2015) The feasibility of extracorporeal membrane oxygenation in the variant airway problems. Ann. Thorac. Cardiovasc. Surg. 21(6), 517-522.

Kunisaki, S. M., Fauza, D. O., Craig, N., Jennings, R. W. (2008) Extracorporeal membrane oxygenation as a bridge to definitive tracheal reconstruction in neonates. J. Pediatr. Surg. 43(5), 800-804.

Lang, G., Ghanim, B., Hötzenecker, K., Klikovits, T., Matilla, J. R., Aigner, C., Taghavi, S., Klepetko, W. (2015) Extracorporeal membrane oxygenation support for complex tracheo-bronchial procedures. Eur. J. Cardiothorac. Surg. 47(2), 250-256.

Marasco, S. F., Lukas, G., McDonald, M., McMillan, J., Ihle, B. (2008) Review of ECMO (extra corporeal membrane oxygenation) support in critically ill adult patients. Heart Lung Circ. 17, S41-S47 (Suppl. 4). 
Netri, K., Votruba, J., Rulíšek, J., Kraus, L., Michálek, P. (2016) Critical airway obstruction during general anaesthesia caused by anterior mediastinal mass managed by ECMO and tracheobronchial stenting. Anest. Intenziv. Med. 27(6), 390-394.

Onozawa, H., Tanaka, T., Takinami, M., Kagaya, S., Tanifuji, Y. (1999) Anesthetic management using extracorporeal circulation of a patient with severe tracheal stenosis by thyroid cancer. Masui 48, 658-661.

Park, A. H., Tunkel, D. E., Park, E., Barnhart, D., Liu, E., Lee, J., Black, R. (2014) Management of complicated airway foreign body aspiration using extracorporeal membrane oxygenation (ECMO). Int. J. Pediatr. Otorhinolaryngol. 78(12), 2319-2321.

Pearson, K. L., McGuire, B. E. (2017) Anaesthesia for laryngo-tracheal surgery, including tubeless field techniques. BJA Educ. 17(7), 242-248.

Pillai, J. B., Suri, R. M. (2008) Coronary artery surgery and extracorporeal circulation: The search for a new standard. J. Cardiothorac. Vasc. Anesth. 22(4), 594-610.

Pinto, C. A., Marcella, S., August, D. A., Holland, B., Kostis, J. B., Demissie, K. (2013) Cardiopulmonary bypass has a modest association with cancer progression: a retrospective cohort study. BMC Cancer 13, 519.

Sablotzki, A., Welters, I., Lehmann, N., Menges, T., Görlach, G., Dehne, M., Hempelmann, G. (1997) Plasma levels of immunoinhibitory cytokines interleukin-10 and transforming growth factor-beta in patients undergoing coronary artery bypass grafting. Eur. J. Cardiothorac. Surg. 11(4), 763-768.

Sanchez-Lorente, D., Iglesias, M., Rodríguez, A., Jungebluth, P., Macchiarini, P. (2012) The pumpless extracorporeal lung membrane provides complete respiratory support during complex airway reconstructions without inducing cellular trauma or a coagulatory and inflammatory response. J. Thorac. Cardiovasc. Surg. 144(2), 425-430.

Schieren, M., Bohmer, A., Dusse, F., Koryllos, A., Wappler, F., Defosse, J. (2017) New approaches to airway management in tracheal resections - A systematic review and meta-analysis. J. Cardiothorac. Vasc. Anesth. 31(4), 1351-1358.

Sen, A., Callisen, H. E., Alwardt, C. M., Larson, J. S., Lowell, A. A., Libricz, S. L., Tarwade, P., Patel, B. M., Ramakrishna, H. (2016) Adult venovenous extracorporeal membrane oxygenation for severe respiratory failure: Current status and future perspectives. Ann. Card. Anaesth. 19(1), 97-111.

Shao, Y., Shen, M., Ding, Z., Liang, Y., Zhang, S. (2009) Extracorporeal membrane oxygenation-assisted resection of goiter causing severe extrinsic airway compression. Ann. Thorac. Surg. 88(2), 659-661.

Sivitanidis, E., Tosson, R., Wiebalck, A., Laczkovics, A. (1999) Combination of extracorporeal membrane oxygenation (ECMO) and pulmonary lavage in a patient with pulmonary alveolar proteinosis. Eur. J. Cardiothorac. Surg. 15(3), 370-372.

Slinger, P., Karsli, C. (2007) Management of the patient with a large anterior mediastinal mass: recurring myths. Curr. Opin. Anaesthesiol. 20(1), 1-3.

Smith, I. J., Sidebotham, D. A., McGeorge, A. D., Dorman, E. B., Wilsher, M. L., Kolbe, J. (2009) Use of extracorporeal membrane oxygenation during resection of tracheal papillomatosis. Anesthesiology 110(2), 427-429.

Tempe, D. K., Arya, R., Dubey, S., Khanna, S., Tomar, A. S., Grover, V., Nigam, M., Makwane, U. K. (2001) Mediastinal mass resection: Femorofemoral cardiopulmonary bypass before induction of anesthesia in the management of airway obstruction. J. Cardiothorac. Vasc. Anesth. 15(2), 233-236.

Tyagi, I., Goyal, A., Syal, R., Agarwal, S. K., Tewari, P. (2006) Emergency cardiopulmonary bypass for impassable airway. J. Laryngol. Otol. 120(8), 687-690.

Vohra, H. A., Whistance, R., Modi, A., Ohri, S. K. (2009) The inflammatory response to miniaturised extracorporeal circulation: a review of the literature. Mediators Inflamm. 2009, 707042.

Walles, T. (2007) Clinical experience with the iLA Membrane Ventilator pumpless extracorporeal lung-assist device. Expert Rev. Med. Devices 4, 297-305. 
Wiebe, K., Baraki, H., Macchiarini, P., Haverich, A. (2006) Extended pulmonary resections of advanced thoracic malignancies with support of cardiopulmonary bypass. Eur. J. Cardiothorac. Surg. 29(4), 571-578.

Willms, D. C., Mendez, R., Norman, V., Chammas, J. H. (2012) Emergency bedside extracorporeal membrane oxygenation for rescue of acute tracheal obstruction. Respir. Care 57(4), 646-649.

Woods, F. M., Neptune, W. B., Palatchi, A. (1961) Resection of the carina and main-stem bronchi with the use of extracorporeal circulation. N. Engl. J. Med. 264, 492-494.

Worku, B., De Bois, W., Sobol, I., Gulkarov, I., Horn, E. M., Salemi, A. (2015) Extracorporeal membrane oxygenation as a bridge through chemotherapy in B-cell lymphoma. J. Extra Corpor. Technol. 47(1), 52-54.

Yuan, K. C., Fang, J. F., Chen, M. F. (2008) Treatment of endobronchial hemorrhage after blunt chest trauma with extracorporeal membrane oxygenation (ECMO). J. Trauma 65(5), 1151-1154.

Pořízka M.; Michálek P.; Votruba J.; Abdelmalak B. B. 